\title{
Third-World Astronomy Network
}

\author{
Jayant V. Narlikar \\ Inter-University Centre for Astronomy and Astrophysics, Pune 411007, \\ India.e-mail: jvn@iucaa.ernet.in
}

\begin{abstract}
Several developing countries of the Third World have been actively interested in astronomy, as is evidenced by the membership of the IAU. The enthusiasm of individual astronomers from these countries is, however, not matched by the resources available to them to pursue their interest in astronomy, in teaching as well as research, at an abovethreshold level. Major problems requiring solutions are (i) isolation from the mainstream work, which leads to research work which is not quite relevant or realistic, and to teaching based on outdated knowledge; (ii) lack of financial resources, leading to shortage of books and journals in the library, insufficient computing power, out-of-date instruments, as well as inability to participate in essential activities like schools, workshops, and major international conferences and symposia; and (iii) lack of handson experience with state-of-the-art instrumentation that often leads to good scientists being turned away from astronomical observations towards abstract theories.

Experience of the International Centre for Theoretical Physics at Trieste, Italy and of the inter-university centres in India, like the IUCAA at Pune, has shown that limited resources can be made to go a long way by sharing, networking and intelligent use of communications technology. Based on the above experience, this proposal envisages setting up a Third World Astronomy Network (TWAN) under the auspices of the IAU, within the wider ICSU-umbrella with support from the UNESCO as well as participating nations. The TWAN will operate with a few key institutions as local nodal points of a wide network. The objectives of the proposed TWAN and the role of the Nodal Institutions (NIs) are spelled out in this proposal, along with the budgetary support required.
\end{abstract}

\section{Introduction}

The Third-World Astronomy Network (TWAN) is a proposal to bring together astronomy institutions and groups of scientists working in astronomy and astrophysics (A\&A) in Third-World countries (TWCs) under one umbrella, so that they can pool their limited resources and improve their work output through several collaborative ventures. The extraordinary progress of information technology makes it possible to translate this idea into reality through networking with a relatively modest investnment. 
What problems will such a network hope to solve? One may identify three main problems faced by astronomers from the Third-World countries:

(1) The field of A\&A is advancing very rapidly and it is very difficult to keep pace with it, even under the best of conditions. Isolation from these developments is the main difficulty faced by astronomers from the TWCs. Not knowing where the subject has reached today, their perception is largely based on old literature, with the result that the research problems they undertake to solve are either already solved or are no longer relevant. Similarly, the teaching of A\&A in the TWCs can be based on outdated curricula and textbooks. So the students doing Ph.D.s enter the field with inadequate foundations.

(2) The priorities for public spending in the typical TWC are rightly oriented towards providing and upgrading basic amenities like water, food and shelter. Basic research figures on the country's agenda, if at all, very low down the list. And amongst the basic sciences astronomy is not seen as addressing downto-earth problems. So funding for A\&A research, teaching and development (RTD) is either nonexistent or at a very low level. Further, even if there are international pedagogical activities like summer/winter schools, research workshops or conferences presenting reviews of state-of-the-art work, the scientists from TWCs are not able to participate in these for lack of funds.

(3) The lack of access to good observing facilities or usage of good instruments and software for observing and data-analysis deters would-be observers from the TWCs from observational astronomy, orienting them towards abstract theory which demands relatively less expensive and elaborate infrastructure. Very often therefore it is seen that this "by default" recourse to theoretical research produces substandard papers. Given suitable opportunities of observations and/or data analysis, the same people could have produced much better science.

Despite these difficulties many TWCs are members of the IAU and their members make valuable contributions to its activities. It is therefore all the more important that the international community of astronomers comes forward to discuss and resolve these difficulties.

There is no foreseeable change in the economic circumstances of the TWCs, or their attitudes and priorities, to raise expectations of a sea change in the above situation. As the International Astronomical Union is the acknowledged world body for astronomy, it provides the right forum to discuss these issues. Moreover, with its established reputation and human resources, it is the ideal body to initiate developments to improve the situation. Which is why I am raising this proposal at the Special Session organized to discuss the state of astronomy in developing countries.

The bottom line of what I wish to propose is this: even with limited resources, one can make a beginning by setting up a network linking TWCs to harness the latest available tools of information technology to upgrade the state of $A \& A$ in these countries. 


\section{The IT Revolution}

The key to resolving the three problems mentioned above lies in the transmission, reception and processing of information. The last decade has seen a remarkable growth of information technology. An example of how this helps can be seen by an episode from my own personal experience, a couple of years ago.

Working in Pune, India, I was then writing a popular book to be entitled Seven Wonders of the Cosmos for publication by the Cambridge University Press, Cambridge. My publisher informed me that a photograph that I had sent of the 300-m dish at Arecibo, for inclusion in the book was not of good quality and asked if I could send a better image. I sent an e-mail requesting a friend in the Arecibo Observatory to send a photograph. He readily obliged by sending the picture file to the publisher electronically. The entire process took no longer than twenty four hours. The cost? Hardly anything. In the old days one would have had all this correspondence by air-mail (now called snail-mail!) between Cambridge-Pune-Arecibo-Cambridge, taking at least about a month.

The important thing about this example is that it has by now ceased to become extraordinary. Transmission of manuscripts and pictures is fast becoming routine, the only limitation being access to sufficient bandwidth.

So can we not harness this quiet but sure revolution towards improving the RTD conditions in the TWCs?

\section{Networking}

Networking holds the key to this approach. Let me give a few examples, from which we can learn something.

In India libraries of eight A\&A institutions have come together under a scheme called FORSA (FOrum for Resource Sharing in Astronomy). These are research institutes and observatories. These libraries share their resources through exchange of information, abstracts of journals, books on inter-library loan, and, more importantly, sharing research journals. Some journals offer special deals for more electronic copies, if one set of hard copy is subscribed to by one library.

My own centre, the Inter-University Centre for Astronomy and Astrophysics (IUCAA) was set up to help upgrade the RTD in Indian universities. We have set up a data centre containing astronomical information, catalogues, etc., a mirror site for the Strasbourg Data Centre, and wide enough bandwidth to access data centres abroad. This facility is made use of by our users, the faculty and students from universities.

In this context the proposal originating in the U.S.A. for a "Virtual Observatory" is very instructive. It is argued in this proposal that archival data from various observations can generate good science through analysis and that they be made available in such a virtual observatory. Thus even without access to world class observatories, one may still hope to do good astronomy from their data.

To show that networking can help also at the other end of the spectrum, for bringing astronomy to schoolchildren, let me narrate another experience at IUCAA. We have set up a link with a 0.4-m telescope at the Mt Wilson Obser- 
vatory in Southern California. Through this link the telescope can be operated and images taken from Pune, at the other side of the globe. We had sessions of this kind with schoolchildren who had come for a summer programme at IUCAA. Because of the 12.5 hours time difference, these children could operate the telescope in broad daylight, at 2. 30 p.m.!

Perhaps I should mention the pioneering work of the late Professor Abdus Salam of Pakistan, the Nobel prize winning particle physicist, in setting up the International Centre for Theoretical Physics in Trieste more than three decades ago. Long before the IT revolution started, the ICTP had been playing a nodal role in bringing together physicists from TWCs for their individual research as well as for pedagogical activities. The Third-World Academy of Sciences which funds collaborations from TWCs was also set up by Professor Salam.

\section{TWAN}

With these examples, it is clear that physical separation from the scene of activity is no longer a prime concern; information can be exchanged across physical barriers, and it can be processed to the benefit of astronmy. Using the available technology today (with of course, future improvements) one can develop the idea of TWAN, a Third-World network in astronomy along the following lines.

A few institutions in participating TWCs are identified as nodes of this network. They will ensure that they have certain basic minimum infrastructure which helps promote RTD activities. These could include, for example, fast e-mail and data-transfer facilities, mirror sites of some data centres, electronic access to any virtual observatory, access to astronomy preprint sites, etc. Each nodal institution (NI) will serve the academic community in its zone which may include more than one country to begin with.

Apart from the "passive" mode of making available access to data, there should be the more active role that each NI may be called upon to play. It should organize schools, workshops, teacher-refresher programmes, etc., for students, researchers and teachers in its region. There can be an instrument-making laboratory which encourages scientists from the TWCs to come and build their own modest level instruments under expert guidance, including a telescope making facility for amateur astronomers.

There is a third mode, the "catalytic mode" in which the NI may help a Third-World scientist to submit an observational proposal at a major facility in the world, also providing a travel grant if the proposal is accepted.

I should stress that the IUCAA at Pune is playing all three roles for the Indian university community ever since its foundation in 1989. It is perhaps symptomatic that the IUCAA was set up just at the time that IT was beginning to grow; and so we have been able to take the advantages of the IT revolution as it unfolded.

\section{Funds}

Now the last but not the least: what funds will be needed to set up the network and to keep it growing? Where can they be generated? Here are some ideas. 
The requirements of the network begin with the provision of adequate infrastructure at each nodal institution for data transmission and storage. Thus adequate server capacity and sufficient band-width for data transmission will be required. Some NIs may already have an infrastructure for their own needs. But it may have to be upgraded to take on the additional responsibility of the nodal role. Some project staff may have to be appointed for running and maintaining the facility. Since the job market lures the talented to commercial ventures, it may not be desirable to have permanent appointments, but one may rather concentrate on a floating population of computer-scientists/engineers who may like to take on the job for 2-3 years to pick up experience.

If there are any protocol/royalty charges to be paid for creating the mirror sites of data centres, provision may have to be made for these amounts. The IAU may also request these charges to be waived, considering the use these centres are to be put to.

Some provision may have to be made for travel/subsistence grants for funding guest-observing visits of Third-World astronomers to major observing sites in the world.

The pedagogical activities of each nodal centre will need financial support, mainly in the form of travel of the lecturers and participants. The former may have to be drawn from the advanced countries, but the latter will be limited to the region. It is expected that each centre will have adequate housing facility for hosting such meets. If not, some initial expense will be needed to set up these.

As the information technology advances further, the travel costs will reduce. Thus remote operating of international telescopes, video lecturing with audience participation, e-mail talks between collaborating scientists across the globe will make it easier and easier to improve the interactive role of TWAN. But a beginning has to be made - sooner rather than later.

Having listed the major items requiring funds to run the TWAN, I now consider the likely sources of these funds. These could come from a seed-support from the IAU under the ICSU umbrella, a recurring grant from the UNESCO, some support from the Third-World Academy of Sciences (which may be invited to cosponsor TWAN), recurring commitment from each TWC through its adhering organization to the IAU, and of course, from private foundations.

\section{Action Plan}

If the idea of TWAN is found attractive, I suggest that the IAU, through its Commission 46 on the teaching of astronomy, may set up a working group to quantify the details and come up with a working budget. This may then be put to the EC of the IAU for further discussion and possible action.

\section{Discussion}

Schreuder asked which countries are Third-World countries? Kochhar interjected that any country that says it is a Third-World country should be accepted as one. Narlikar said that the Third-World Academy of Sciences has a list which we could adopt. 